\title{
Test Strategies for Blockchain Technology
}

\author{
Soumya ${ }^{1}$, Naresh $E^{1}$, Vijaya Kumar B P $^{1}$ and Ravi B C \\ ${ }^{1}$ Department of Information Science and Engineering, M S Ramaiah \\ Institute of Technology, MSR Nagar, Bengaluru, India \\ ${ }^{2}$ Prayag software Pvt. Ltd Bengaluru, India
}

\begin{abstract}
Blockchain is one of the trending technology. It is a secured distributed public digital ledger that is programmed in order to record the information and keep it securely, the main purpose is to store the data securely and carry out the digital transactions safely. Blockchain technology use cryptography to achieve the security, where the data is encrypted. Blockchain is decentralized and distributed, brings the trust in the data, the data are collected in blocks and each block is connected with other block which makes it a chain hence it is known as blockchain. The main aim in Blockchain technology is to ensure the security to software used in technology, hence the testing phase plays an important role. Testing is mainly done to check block size, chain size, load, security, transaction of data, cryptographical data etc. The main objective of this paper is to ensure the importance of testing the Blockchain technology and different strategies used for testing to increase the security and trust of the technology.
\end{abstract}

KEY WORDS: BLOCKCHAIN, TESTING LIFECYCLE, SMART CONTRACTS, BLOCKCHAIN ORIENTED SOFTWARE, ETHEREUM, GANACHE, HYPERLEDGER COMPOSER.

\section{INTRODUCTION}

Blockchain is an incorruptible, immutable digital ledger of economic transaction that can be programmed to record the values. The Blockchain technology aims in storing the data securely especially for online transactions (Watanabe et al. 2015)and confidentially so no changes can be made once when the data is stored. Blockchain technology was coined in the year 1991 by two great person Stuart Haber and W. Scott Stornetta. In 1992 Bayer, Haber and Stornetta started designing Block-chain technology using Merkle trees which improved the efficiency by collecting the data documents into blocks. The Block-chain was first implemented in a cryptocurrency Bitcoin, as it was best suitable for online transaction and it served as a public

\section{ARTICLE INFORMATION}

*Corresponding Author: soumyablb@gmail.co

Received 15th Oct 2020 Accepted after revision 29th Dec 2020

Print ISSN: 0974-6455 Online ISSN: 2321-4007 CODEN: BBRCBA

Thomson Reuters ISI Web of Science Clarivate Analytics USA and Crossref Indexed Journal

\section{Clarivate
Analytics}

NAAS Journal Score 2020 (4.31)

A Society of Science and Nature Publication,

Bhopal India 2020. All rights reserved.

Online Contents Available at: http//www.bbrc.in/

Doi: $h$ ttp://dx.doi.org/10.21786/bbrc/13.13/39 ledger for all network transaction. The main idea behind the Block-chain technology is that data are stored in a block and once the data is stored it cannot be edited or changed without authorization and these Blocks are linked in series like a chain hence the name 'Blockchain' as shown in the below figure 1 .

Figure 1: Blockchain

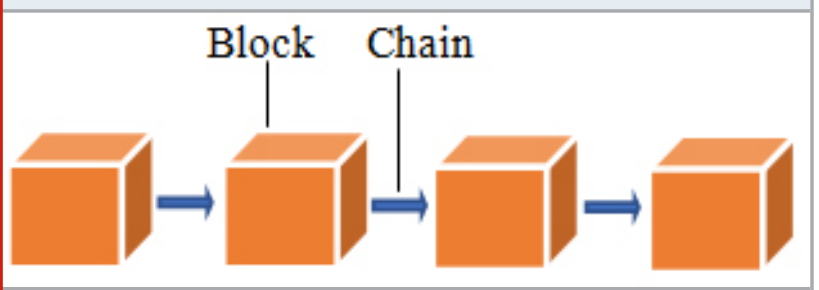

\section{A. The features that make Blockchain technology:}

- It is a decentralized and distributed ledger [2] [3] that can be shared across a large public or peer to peer network. 
- It ensures the trust in data that is the data is stored is such a way that a ledger record the transaction details among the members in a network in an order and each transaction record has a time detail and a unique cryptographic signature.

- Block-chain technology eliminates the intermediaries. For examples there is a transaction of land ownership between two people then a middleman such as lawyer is between two people who will be a proof for maintaining the record details but now on using a Blockchain technology no intermediaries required itself maintain the proof record.

In simple words block chain technology is a security system to maintain records and for economic transactions, it can be explained as if $\mathrm{X}$ change the information stored in block to $Y$ then it is not rewritten instead the change is stored in a new block showing that X changed to $\mathrm{Y}$ with date and time, data in Block-chain are stored in blocks and each blocks contains three main parts Data, Hash and previous Hash. Data is the information that must be stored securely using Blockchain technology. Hash is a unique identity to each block it is like a finger print, it is the one which is responsible for the security of the blocks hash is the address of block (Buterin 2014; Subramani et al. 2020 ), once the block is created a unique hash is calculated if any data in block is modified or changed then hash will be automatically updated.

Previous Hash is one which contain the address of the previous block, and it forms the chain if any previous Hash is updated then the chain is broken that is the link is disconnected and hence proof of work is done to change the hash. Every new block is created by proof of work ad block-chain technology is distributed system if one person adds the block that it is visible to the whole network and everyone in the network can view new block. As Blockchain technology has become efficient technology it is widely used in industries, for tracking the account sessions and maintain the data management solutions. Hence Blockchain technology has many use cases in present years for agriculture and IOT security and privacy (Dorri et al. 2017).

The different testing strategies like functional testing, security testing, smart contracts which is most popularly used testing technique and so on. The different tools like Ethereum, ganache and Hyperledger composer is used for testing, now as the blockchain technology has become very useful and efficient technology it is used widely industries and in agriculture which involves food safety, supply chain etc.

B. Advantages and Disadvantages: The information and transactions are under the user control which is accurate and unique. This is the major advantage of the Blockchain technology that makes the block-chain technology popular. Blockchain does not have any central point of failure and has a capability to withstand the attacks as it is decentralized and distributed system. Interbank transaction can take a day to clear the settlements especially on nonworking hours Blockchain transactions can reduce days to minutes and processed $24^{*} 7$.

1.Lower transaction rate: As third party is eliminated in the Blockchain technology that reduce the transaction fees.

2.High quality data: The data in Blockchain technology is accurate, consistent, complete and timely available.

Blockchain technology is fully towards a decentralized network where every user and operators are fixed to network.

1. Growing technology: As it is a growing technology and it became difficult understand and analyze the new technology and the transaction speed is high, the verification process and data limit are hard to target.

2. Large energy consumption: The blockchain testing is used in wide applications and the network's miners target trillion solution per second for transaction and verifications of data hence it uses the large amount of energy hence large energy is consumed which become as a disadvantage.

II. Literature Survey: The Blockchain technology is a type of data storage where information, transaction details and some additional data of validation are stored, the application that are based on block-chain technology should ensure the data integrity and unique based blockchain system that are trustworthy, which in case of Blockchain oriented software (BOS) is a critical security system. The testing process includes some suites (Norta et al. 2015) they are Block-chain transaction testing, to ensure the security during the transactions ii) Some Traditional testing to securely store the data. There are different tools used for testing the Blockchain technology. The main characteristic of blockchain technology (Li et al. 2017) is that first a transaction is requested by node, and broadcasted to peer-2-peer network then transaction validation is done which involves testing and process is known as smart contracts then verified and blocks are created and tested, then each blocks are verified by block testing and transaction is completed. Testing is done in in different phase with input and by observing the output and comparing it with the expectations. Infosys has issued a while paper that "Assuring the success in block-chain implementation is by engineering quality in verification and validation" (http://www.bcs.org/content/conWebDoc/56020 Infosys Whitepaper).

A Blockchain software project works on blockchain technology as a distributed system, the Bitcoin [10] is the first efficient and popular cryptocurrency that use the blockchain technology. As security in blockchain technology is ensured by the cryptography concepts and the hash are encrypted so that the security can be ensured. To ensure the security of block-chain technology then the different technique was introduced, as there are some tradition methods for testing, but it is not efficient as new technology. This blockchain technology has a 
wide exposure as it is used in cryptocurrency transaction (Yuan \&t Wang 2018), and in a real estate business and so on, as technology is growing up day by day the new tools are emerging for testing purpose to increase the efficiency of software testing that implies the efficiency of the Blockchain technology.

III. Proposed Strategies: In the proposed strategies, there is a brief description on complete software testing lifecycle to test the Blockchain oriented software (BOS) projects and testing involved in different Blockchain applications, as testing is important in part of the software life cycle, testing is important to ensure the quality of application and to test the security in the blockchain technology. In this paper we are discussing about testing life cycle involved in the BOS and different test strategies used for testing such as unit testing, functional testing, security testing, unit testing and a description about the smart contract and the volume of tests, methodology used in testing. The different tools used for testing the blockchain technology are like Ethereum (Porru et al. 2007) which is most commonly used for security testing, Ganache, Hyper ledger composer etc. As block-chain technology is widely used in industries, companies and even for user level, this technology is suitable for the large scale system. Considering one of the case studies "The blockchain technology for agriculture" a brief description on food safety, potential benefits and different testing involved in it is taken as a case study and India is towards launching the blockchain technology for agriculture.

IV. Testing Phases: Though Block-chain technology is a public ledger that is to provide the security to store data and to provide secured online transaction, but to the perfect functionality of technology testing must be done. Blockchain oriented software testing is a testing cycle that is done to synergy between the systems and component in involved in that system and it is a beneficial to Block-chain oriented engineering (Aberdour 2007). The block-chain technology has a different lifecycle for testing the system known as BOS life cycle as shown in figure 2, it mainly involves four different phases they are.

Phase 1 - In this phase the functional workflow of testing is done, and system component is mapped for further testing process this is the first phase of testing.

Phase 2 - This is the second phase in the testing workflow here mapping of blocks is done, prepare the use case outline and verifying the security of the system and the outcome is to prepare the test strategy document.

Phase 3- In third phase testing methodologies are prepared and coverages are estimated, tools are tested, and automation testing is done, use case mapping is done the outcome is that final test strategy and final test case are prepared.

Phase 4- This is the final phase in testing lifecycle and here test execution is done, low level verification, validation of blocks and smart contracts and transactions

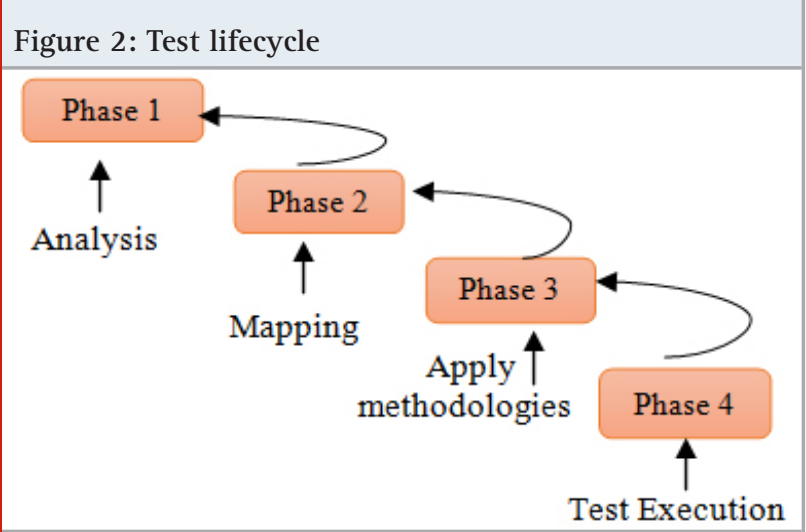

and in this phase third party interface check is done and the outcome is the final report and test results and defect report.

V. Test Strategies For Blockchain: Testing experts test the block-chain technology to ensure the perfectness of the system and to provide a standard technology to the user, as the main aim of Blockchain technology is to provide the security so first the system should be tested to provide the efficient system, there is a separate team to carry out the testing process, some basic testing is done to the system like testing each block, testing the functionality, performance, security etc.

1. Block testing is done to test each block with respect to the information stored in it, the hash present on each block, content of previous hash and so on are tested in the block testing as misplacement of hash may leads to the breakage of the chain, and one of the important challenge is the Block size testing is done on block load.

2. Functional testing is the basic testing in blockchain technology it presents the overall function and operations in the system and it helps to test the business circumstances, effectiveness of the use case scenario and different process involved in system, functional testing also involves the testing of chain size, data transmission, adding of block to the chain and the occurrence of defects are more in this testing.

3. Unit testing is a fundamental testing and an automated testing (Cheon \& Leavens 2002) that is specially used by the developers for testing the code of the software in block-chain technology and check the performance of code at all level of functionality. This testing is always done first because the chances of occurring bug or defects are less and the defect prediction volume is high as it is shown in the Table 1.

4. Integration testing is one of the important testing, integration testing is done to check the collaboration of the component in the system and cohesive of different system and it can be deployed when the system is running parallel and to check the coordination among the system working together, this testing is useful to test when the new block is added to the chain hoe it collaborates with the 
other block.

5. Security testing is a non-functional testing, security testing is mainly done to ensure the security of the system and to check the attack such as virus, malicious etc. security testing is the acknowledged problem (Bau et al. 2010) that is the most important testing some important aspect in security testing are confidentiality, denial of service, availability, integrity. Security testing becomes important to find the identity layer attack which may lead to the expose in transaction, this testing is done in some different phases to ensure the accuracy, and security testing is done to verify the hash of the block, one of the latest and efficient testing done for security purpose is the smart contracts.

6. Performance testing is a non-functional testing, performance testing is done mainly because to test the transaction size and number of transaction, the testing team also focus on the dependent parameters such as network latency, the transaction speed at every node, processing speed, system and user interface, performance testing is made automated to increase the scalability of the blockchain technology.

Table 1. Testing Levels with Volume of tests

\begin{tabular}{|l|c|}
\hline Testing levels & $\begin{array}{c}\text { Volume of } \\
\text { Test Data (\%) }\end{array}$ \\
\hline Unit Testing & 30 \\
\hline Integration Testing & 12 \\
\hline System Testing & 15 \\
\hline Functional Testing & 10 \\
\hline
\end{tabular}

Each testing can be represented in the levels based on the volume of testing for defects, in order it is shown in Table 1, the first is the unit testing it is the fundamental testing that is used to test the coding part which is the fundamental part and the detection of defects are less but expectations are more when compared to other testing and next comes the system testing next Integration testing which is the testing which gets the less defects and next is the functional testing which is fundamental testing where the chances of defect occurrences is more.

A.Smart contracts: Smart contracts are one of the fundamental validation technology that is within the block-chain technology, the main aim if smart contract is to provide the security and reduce the transaction rate when compared to other traditional model, some features that make smart contract unique is (Savelyev 2017) software implementation, increased certainty, self-performance.

Smart contracts are nothing a set of rules that are in the form of programmable format, that are evoked themselves when conditions are met. When smart contracts are introduced to block-chain then it is give an address and contract is notified by its address and condition is checked when it is satisfied smart contract are evoked. For example, if the precondition is transaction to the specific ledger then it under goes some condition that are robust for validation, as smart contracts are similar to the API interphase, as block-chain is distributed smart contact is public functions that can be called by anyone who is registered to that block-chain and any external web API cannot be called. Once the contract is created it cannot be changed, if any defect is found in prediction then new contract must be created, if new is created then previous data has to be reinitialized, even update is not efficient hence smart contract has to be handled carefully (Wöhrer \&t Zdun 2018).

B.Comparison of different testing: The below figure 3 shows the comparison of different testing with defect analysis as series 1 represents the defects introduced in this phase (defects taken for testing) and series2 represents the defects found in this phase (actual defects). It is found that in unit testing the defect taken to test is more, and the actual defects are less but in other testing the defect introduced is less and actual defect are more as represented in below graph figure 3. To find the accurate defects in each testing we use different prediction metrics like Precision, Recall and accuracy, these metrics uses different factors like TP (true positive), FP (false positive), TN (true negative), FN (false negative) (Fawcett 2016). By using these factors we can get the precision as shown in equation(1), where precision gives the quality of the system upon errors, we can find the recall which is calculated as true positive with true positive and false negative as shown in equation (2) and it gives defect in the system, then we find the Accuracy which is calculated as true positive and true negative with all the total entity like true positive, true negative, false positive, false negative as shown in equation (3) and it is used to find the accuracy of the system.

Precision=TP / (TP+FP)

Recall $=\mathrm{TP} /(\mathrm{TP}+\mathrm{FN})$

Accuracy $=(\mathrm{TP}+\mathrm{TN}) /(\mathrm{TP}+\mathrm{TN}+\mathrm{FP}+\mathrm{FN})$

\section{Figure 3: Comparison of testing}

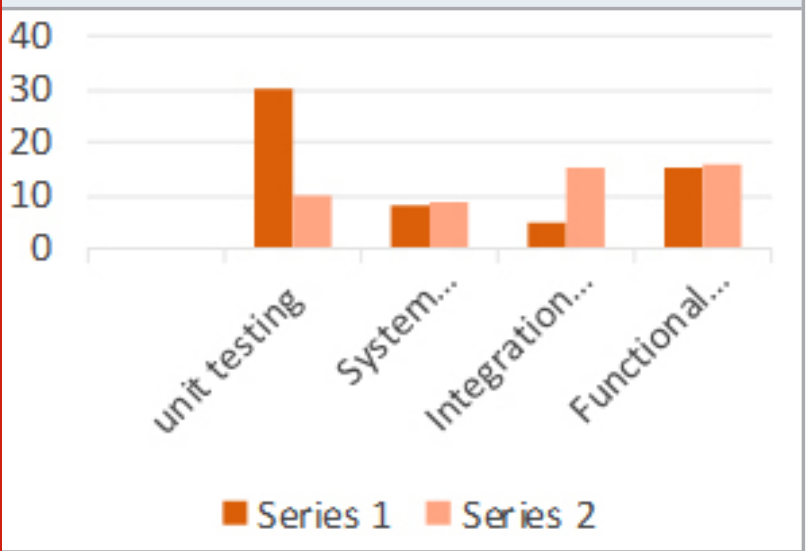


Using these methods, the defect prediction is done, and different testing is done using this prediction methods as different testing is carried out for different purpose, but each testing has its own role in testing phase, the different volume of data is collected for different testing.

VI. Tools Used For Block-Chain Testing: Blockchain is a distributed peer-to-peer and client server model (Lewis 2016)hence testing is a necessary, Testing is mainly done to increase the performance of system and to check the defects or bugs in the system, to test a system there are different testing technique and to do testing there are different tools example Ethereum, Ganache, Hyperledger composer.

1. Ethereum is one of the flexible tool to build a blockchain app, it is the combination of both development and testing tool, where the state of system is mapped between address and account state (Wood 2018), it supports all type of computations including the loops, Ethereum tester is reliable for functional testing, security testing, smart contracts and other block-chain testing.

2. Ganache is also a reliable tool used to test the Ethereum contracts, it creates a reliable block-chain that allow user to use multiple accounts for testing, Ganache is used in collaboration with the Ethereum for the security purpose, Ganache set up to 10 default Ethereum address with each primary key and load them in the initial phase, Ganache is mainly used in private Block-chain.

3. Hyperledger composer is a tool for testing, and is an open source with collaborative effort that is used in usage of industrial Blockchain technology, it was introduced by Linux foundation but it lacks most of block-chain testing features, hence it is useful to test the app before it is being launched, this tool is mainly useful in interactive and automated unit testing.

Case study for Block-chain: Considering a case study 'The Blockchain technology for agriculture and food chain supply' agriculture is the backbone of the country, many families in our country are dependent on agriculture so to provide a required provisions and requirements for agriculture and formers. The essence of Block-chain in agriculture is to maintain the data management solution (Patil et al. 2017). Blockchain technology is used in the agriculture in the fields of food safety, food security, potential benefit and former support, in brief it can be described as.

Food safety is one of the important feature in agriculture and where the food has to be safely preserved for the usage, here the block-chain technology can be used to store the documents of food grown and proper usage of pesticides everything can be stored to follow the rules in this model security testing place an important role it should be done correctly.

Food security is also one of the important issue as crops grown are lost due to smuggling and there is no proper guide so again block-chain technology should be used to maintain the record of crops grown. Potential benefits is one which is in consider with former, now a days formers are not getting proper benefit, government has declared many facilities but it is not reaching to formers so to solve this problem there should be direct transaction between government and former, and government should set a proper market price to grown food and maintain the record which helps the former to keep record.

Former support it is a common problem in villages that are faced by the formers, there is no proper support and guidance for the formers as government is providing many facilities to the former and agriculture by they are not aware of it due to improper communication and improper documentation, hence block-chain technology solves this problem to maintain the record that information is reached to formers and they have replied to it and make the whole system digitalize.

\section{CONCLUSION}

To ensure the efficiency of the Blockchain technology, the software inside the technology should work efficiently and should be tested hence testing is also important aspect, and BOS phases of testing are specified above and every testing here involves this process, and different testing technique used for testing and tools used for testing, smart contract which is the efficient and unique way of testing block-chain which is widely used in industries and smart contract has a great features, when compared to overall testing smart contract is used widely, the comparison of testing with defect introduced to defect found are estimated in graph, the main purpose of block-chain is to provide the safe transaction hence it has a wide application now in India there is a plan to incorporate it into agriculture sector, hence we can conclude that testing is an important strategy to Blockchain technology to make it efficient.

\section{REFERENCES}

Hiroki Watanabe, Shigeru Fujimura, "Blockchain Contract: A Complete Consensus using Blockchain”, 2015 IEEE 4th Global Conference on Consumer Electronics (GCCE).

S.A.A.: Blockchain Ready Manufacturing Supply Chain Using Distributed Ledger. Int. J. Res. Eng. Technol. 05, 1-10 (2016).

D. Puthal, N. Malik, S. Mohanty, E. Kougianos, and C. Yang, "The Blockchain as A Decentralized Security Framework”, IEEE Consumer Electronics Magazine, Vol. 7, No. 2, pp. 18--21, 2018.

V. Buterin, "A next-generation smart contract and decentralized application platform," white paper, 2014.

Subramani, P., Rajendran, G.B., Sengupta, J., Pérez de Prado, R. and Divakarachari, P.B., 2020. A Block BiDiagonalization-Based Pre-Coding for Indoor Multiple- 
Input-Multiple-Output-Visible Light Communication System. Energies, 13(13), p.3466.

A. Dorri, S. Kanhere, R. Jurdak, and P. Gauravaram "Blockchain for IoT Security and Privacy: The Case Study of a Smart Home", in Proceedings of the IEEE International Conference on Pervasive Computing and Communications Workshops, pp. 618-623, 2017.

Alex Norta et.al, "Conflict-Resolution Lifecycles for Governed Decentralized Autonomous Organization Collaboration", St. Petersburg, Russian Federation ำ 2015 ACM.

X. Li, P. Jiang, T. Chen, X. Luo, and Q. Wen, "A Survey on the Security of Blockchain Systems", Future Generation Computer Systems, 2017.

Testing of Blockchain: http://www.bcs.org/content/ conWebDoc/56020 Infosys Whitepaper

Kombe, Cleverence \&t Manyilizu, Majuto \&t Mvuma, A. (2017). Design of Land Administration and Title Registration Model Based on Blockchain Technology. Journal of Information Engineering and Applications. 7. 8-15.

Yuan, Y., \& Wang, F.-Y. (2018). Blockchain and cryptocurrencies: Model, techniques, and applications. IEEE Transactions on Systems, Man, and Cybernetics: Systems, 48(9), 1421-1428.

Simone Porru et.al, 2017. Blockchain-oriented Software Engineering: Challenges and New Directions", 39th IEEE international conference on Software Engineering Companion.

M. Aberdour, "Achieving quality in open-source software," IEEE software, vol. 24, no. 1, pp. 58-64, 2007.

Y. Cheon and G. T. Leavens. A simple and practical approach to unit testing: The JML and JUnit way. In Proceedings of the 16th European Conference on ObjectOriented Programming, ECOOP '02, pages 231- 255. Springer-Verlag, 2002.

J. Bau, E. Bursztein, D. Gupta, and J. Mitchell. State of the art: Automated black-box web application vulnerability testing. In Security and Privacy (SP), 2010 IEEE Symposium on, pages 332-345. IEEE, 2010

A. Savelyev, "Contract law 2.0: 'Smart' contracts as the beginning of the end of classic contract law," Information \& Communications Technology Law, vol. 26, no. 2, 2017, pp 116-134.

M. Wöhrer and U. Zdun, "Smart contracts: Security patterns in the Ethereum ecosystem and solidity," Proceedings of the International Workshop on Blockchain Oriented Software Engineering, 2018, pp. 2- 8.

T. Fawcett. An introduction to ROC analysis. Pattern Recognition Letters, 27(8):861-874, June 2006.

A Lewis, "So You Want to Use a Blockchain for That?" Jul. 2016.

G. Wood, "Ethereum: a secure decentralised generalised transaction ledger, Byzantium version," 2018.

Patil, A. S., Tama, B. A., Park, Y., \&t Rhee, K. H. (2017). A Framework for Blockchain Based Secure Smart Green House Farming. Advances in Computer Science and Ubiquitous Computing (pp. 1162-1167). Singapore: Springer. 